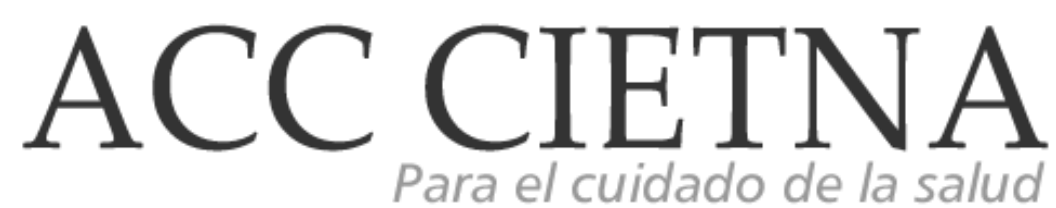

https://doi.org/10.35383/cietna.v7i1.364

ENSAYO

\title{
Interacción durante el cuidado enfermera-persona en estado de embriaguez
}

\author{
Acevedo Figueroa Lucila ${ }^{1}$, Álvarez Aguirre Alicia ${ }^{2}$, Casique Casique Leticia ${ }^{3}$, Hernández \\ Rodríguez Veronica Margarita 4
}

\begin{tabular}{ll}
\hline INFORMACIÓN DEL ARTÍCULO & RESUMEN \\
\hline Historia del artículo: &
\end{tabular}

Recibido el 08 de junio de 2020

Aceptado el 28 de junio de 2020

El cuidado de enfermería se fundamenta en la interacción que

Palabras clave:

Interacción

establece la persona con el equipo de enfermería en diferentes

Cuidado de enfermería

escenarios; el presente ensayo corresponde a la interacción

Persona en estado de embriaguez enfermera-persona en estado de embriaguez, en un escenario hospitalario, en donde el ejercicio de la práctica profesional de enfermería es lo que articula los cuidados, en el área asistencial y tecnológica. Durante la interacción que surge al proporcionar el cuidado, los sentimientos cumplen una función particular, es en este momento donde enfermería debe poseer algunos atributos indispensables como la sensibilidad humana para estar disponible a las solicitudes y necesidades de la persona en estado de embriaguez la cual posee características propias que pueden ser profundas alteraciones en la percepción, en el curso del pensamiento, en la sensibilidad, en el juicio, en la esfera afectiva. Podemos ultimar que la interacción es una relación especial, no solo un evento que acontece en ambas personas, el acto mismo del cuidado surge en la interacción en el sujeto mismo, pero no solo como instinto sino como creencia de la propia existencia. La interacción durante el cuidado en un contexto hospitalario es un acto de vida que beneficia a ambas personas, permitiendo crecer juntas.

\footnotetext{
'Estudiante de Doctorado en Ciencias de Enfermería en el Campus Celaya Salvatierra Universidad de Guanajuato. Enfermera en el Hospital General de Taxco de Alarcón Guerrero, México. E-mail: acevedo_lucy@hotmail.com. ORCID. https://orcid.org/0000-0001-6471-684X

2 Doctora en Ciencias de Enfermería. Docente de la Universidad de Guanajuato en el Campus Celaya Salvatierra, México. Email: alvarez_ali@yahoo.com.mx. ORCID: https://orcid.org/0000-0001-5538-7634

3 Doctora en Ciencias de Enfermería. Docente de la Universidad de Guanajuato en el Campus Celaya Salvatierra, México. Email: leticiacc_2004@yahoo.com.mx. ORCID: https://orcid.org/0000-0002-0532-4819

${ }_{4}$ Doctora en Investigación Educativa. Docente de la Facultad de Enfermería de la Universidad Autónoma de Querétaro, México. Email: covetojo@yahoo.com. ORCID: https://orcid.org/0000-0002-5795-0483
} 
Interaction during nurse care to a person in state of intoxication

ABSTRACT

Keywords:

Interaction

Nursing care

Person in state of intoxication
Nursing care is based on the interaction that the person establishes with the nursing team in different settings; This essay concerns the nurse-person lin state of intoxication interaction in a hospital setting, where the practice of professional nursing is what articulates care, in the healthcare and technological area. During the interaction that arises when providing care, feelings fulfill a particular function, it is at this time that nursing must possess some indispensable attributes such as human sensitivity to be available to the requests and needs of the person with alcohol consumption who has own characteristics that can be profound alterations in perception, in the course of thought, in sensitivity, in judgment, in the affective sphere. We can conclude that the interaction is a special relationship, not only an event that occurs in both people, the very act of caring arises in the interaction in the subject himself, but not only as instinct but as a belief in his own existence. Interaction during care in a hospital context is an act of life that benefits both people, allowing them to grow together.

\section{Introducción}

Enfermería fundamenta su práctica profesional en una relación enfermera-paciente en la cual el profesional de enfermería es un instrumento terapéutico y las interacciones se efectúan para lograr un objetivo o transacción. La comunicación y el dialogo se dan como procesos recíprocos que se proporcionan en situaciones de encuentros o desencuentros por los que se comunican pensamientos, sentimientos $y$ actitudes, se comparten subjetividades, lo que permite ver al cuidado como una labor humanitaria que facilita el crecimiento de cada uno en un proceso que difiere en cada caso ${ }^{1}$. La práctica de enfermería se ha realizado en sus primicias bajo el modelo biomédico, desde el enfoque determinista, ha desarrollado la base del conocimiento y la práctica de la enfermería conduciendo a un reduccionismo y promoviendo el cuerpo biológico que es atendido por enfermería ${ }^{2}$.
Favorablemente en las últimas décadas enfermería ha cambiado, articulando valores que enfatizan procesos humanos de cuidado, ha involucrado valores que reconocen también la naturaleza racional y la complejidad de la experiencia humana.

En el contexto hospitalario la interacción de enfermería con la persona en estado de embriaguez surge en servicios de urgencias y hospitalización, esta persona se caracteriza por un estado transitorio de falta de coordinación motora y un oscurecimiento de la conciencia provocado por la intoxicación de alcohol, la cual depende del grado de etanol que haya ingerido dependiendo la bebida consumida ${ }^{3}$, asimismo las personas en estado de embriaguez pueden estar con mayor frecuencia en las instituciones hospitalarias por complicaciones derivadas de traumatismos, violencia familiar o enfermedades relacionadas con el consumo de alcohol. En este escenario las interacciones se realizan también con diferentes actores, negociando el cuidado 
cuyo objetivo principal es brindarlo de manera holística ${ }^{4}$.

En las interacciones humanas hay un contacto moral para dar un trato equitativo justo $y$ respetuoso, también hay un apego una relación afectiva que responde a la solicitud de cuidado para satisfacer las necesidades, la interacción entre es asimétrica y proviene de la de la experiencia de la vulnerabilidad presente solo les separa la potencia y la intensidad de la vulnerabilidad que son las que establecen la diferencia el que proporciona el cuidado y el que es cuidado 5 .

Se puede considerar que durante la interacción con la persona en estado de embriaguez pueden emerger las características propias de la persona como la necesidad de ser protegido, solicitar apoyo y afecto, sentirse fácilmente desamparado e inseguro y a depender aun económicamente de los demás; a esta dependencia se agrega una actitud indolente, de autocastigo, a humillarse y a sentirse con frecuencia criticado y castigado. estos tres rasgos (dependencia, pasividad, y culpa), junto a pesimismo, hostilidad, aislamiento, soledad, timidez, esquivez, indiferencia, desapego, tendencia a la depresión, corresponden a las características de esta persona6. En el presente ensayo se aborda la interacción teoría-modelo, la evidencia y experiencia humana durante el cuidado enfermera-persona en estado de embriaguez en un contexto hospitalario.

\section{Desarrollo}

Diversos autores que han abordado el concepto de interacción como elemento fundamental en el cuidado de enfermería asocian en sus teorías algunas características como empatía, respeto, responsabilidad, comunicación, reciprocidad, relación de ayuda, compasión y apoyo, como se muestra a continuación:
Kash, considera que la enfermería es un proceso de interacción social mediante el que la enfermera induce una mejora en el estado de salud de los pacientes como resultado del diálogo terapéutico; menciona que la enfermera (o) es una estratega de la comunicación que controla el comportamiento interpersonal para aumentar la probabilidad de alcanzar objetivos intentando satisfacer las necesidades de comunicación de un paciente realizando diversas funciones ${ }^{7}$.

También Lamongtane, plantea que la relación de ayuda es una herramienta en el proceso de enfermería y es una condición necesaria para cuidar verdaderamente y acompañar al paciente durante todo su proceso de recuperación o de acercamiento a la muerte con dignidad y autenticidad $^{8}$.

En igual forma King Imogene, considera que "la interacción es un proceso de percepción y comunicación entre una persona y el entorno o entre una persona y otra que se manifiesta por conductas verbales dirigidas hacia un objetivo" 9 .

En ese mismo contexto Gala, menciona que una enfermera socialmente hábil, es capaz de interactuar y comunicarse eficazmente entablando una interacción con sus pacientes y demás profesionales, convencida de la importancia de ellos ${ }^{10}$.

Así mismo Pike, menciona que la empatía es la clave para la comunicación del interés y apoyo al paciente; se puede establecer una relación de empatía mirando al mundo desde el punto de vista del paciente y sintiendo el mundo del paciente ${ }^{11}$.

Igualmente, Bizier, alude que como la enfermera está habitualmente en contacto con personas que tienen necesidad de ayuda, es importante que establezca relaciones de calidad, de tal manera que permita al paciente expresarse abiertamente sin tener miedo a ser juzgado ni rechazado ${ }^{12}$. 
De forma similar Joyce Travelbee considera que la enfermera en la relación persona a persona, vive la socialización como la transmisión de saberes y valores, como adaptación al medio, costumbres y normas en el encuentro existencial dentro de la interacción de un yo con un tú y un nosotros en condiciones de igualdad, respeto y afecto $^{13}$. Modelo como el de Joyce Travelbee ofrecen un marco de referencia sobre las relaciones entre las personas, la síntesis exclusiva de sus ideas hace reflexionar sobre la necesidad de desarrollar el encuentro en una relación terapéutica (Figura 1).

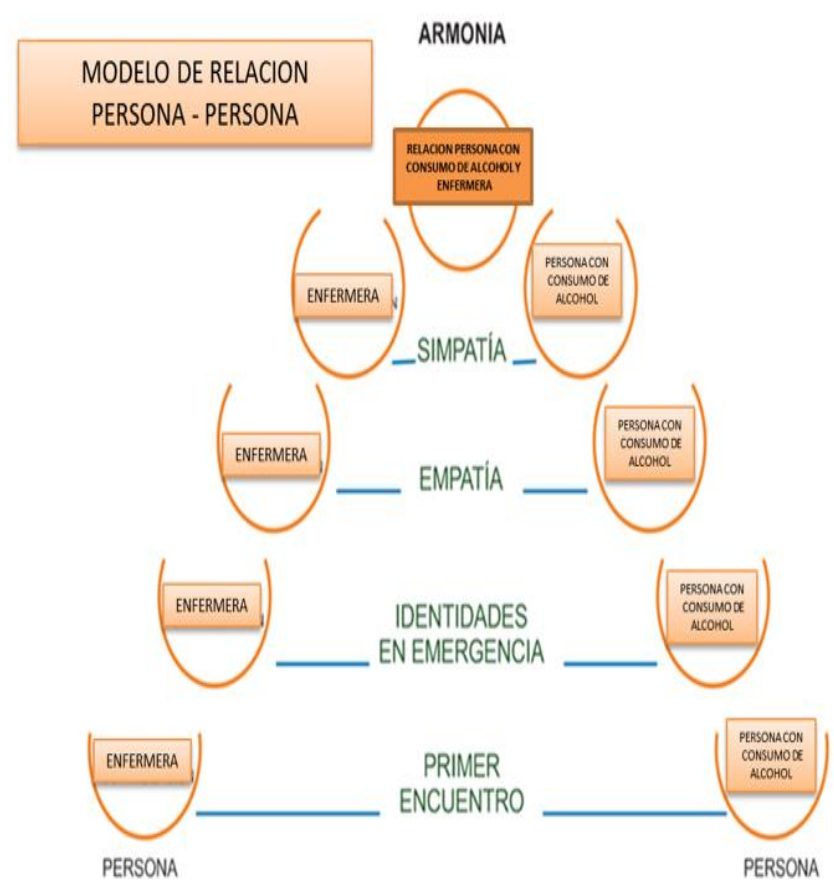

Figura 1. Modelo de Joyce Travelbee, enfermerapersona en estado de embriaguez.

En este mismo contexto Henderson, plantea que el proceso de cuidado de enfermería no puede efectuarse sin un enfoque de relación de ayuda con el paciente; es necesario que se establezca una relación significativa (interacción) en la que la enfermera debe adoptar actitudes de respeto, comprensión, empatía, autenticidad y de consideración positiva ${ }^{14}$.

De manera análoga Martin Heidegger menciona que la relación del Dasein con el cuidado, es el estar ahí, en este caso en el mundo de la enfermera y la persona, en la vida de sí misma, donde reconoce su ser, lo que quiere para su existencia en relación con la salud y la enfermedad.

Es así como la enfermera (o) se reconoce como un ser ahí que en interacción con la otra persona, reflexiona sobre su contexto vivencial e interpreta la realidad de la práctica, condición esencial para la comprensión del ser cuidado, el cual en su abordaje más amplio consiste en una forma de ser y estar con otros posee características existenciales y contextuales (modo de ser) y (estar con), de tal forma que el cuidado se visualiza en 2 dimensiones: fenomenológica y holística, es decir, entender los significados que la persona le confiere a sus experiencias en interacción con la enfermera (o) quien la impulsa para encontrar su ser ahí o en su caso le provee cuidados de información, de mantenimiento de la salud o de curación como un estímulo para lograr el cuidado de si, de igual forma, en la persona con consumo de alcohol aumenta el deseo de existir el poder de descubrir el mundo de comprenderlo y de centrarse en el como persona, sin señalamientos, en consecuencia es un acto de vida que se desarrolla en el contexto de la existencia humana ${ }^{15}$.

La evidencia científica muestra la importancia de la interacción en personas con estado de embriaguez por mencionar: El estudio sobre las experiencias del personal de enfermería con pacientes hospitalizados por abuso de alcohol, revelo que actitudes negativas por ejemplo el rechazo a estos pacientes condiciona la atención, sin embargo, el antecedente de un familiar que consume alcohol se asocia a una mayor empatía y disposición al cuidado de estas personas por el personal de enfermería ${ }^{16}$.

La investigación acerca de la percepción, conocimientos y actitudes de las enfermeras de urgencias y salud mental frente al alcoholismo y otras drogodependencias, reporto el 75\% había tenido dificultades con los pacientes entre los 
aspectos que dificultaban la atención eran: falta de conocimientos, actitud del propio profesional y percepción social, falta de habilidades en la interacción, experiencias previas, organización y tipo de servicio donde trabajaba y opiniones personales, el estudio concluye que la enfermera percibe al paciente alcohólico como una persona enferma que necesita terapia ${ }^{17}$.

En el contexto de la experiencia humana Mélich en su libro ética de la compasión, aporta que desde el nacimiento habitamos un espaciotiempo que no elegimos; el mundo que empezamos a conocer ya ha sido habitado por otros antes que nosotros y no tenemos control sobre él. Nuestra primera condición es de vulnerabilidad, en donde nos encontramos amenazados por procesos de caotización: el azar, la soledad, la culpa, la nostalgia, el sufrimiento, donde la interacción se veda.

Así mismo Mélich menciona la frase de Elías Canetti en uno de sus ensayos Masa y poder: Nada teme el hombre más que ser tocado por lo desconocido, en un contexto hospitalario todo puede ser desconocido así se haya permanecido en varias ocasiones, las personas que consumen alcohol son "seres-en- falta", como todas las personas y para ello necesitan de los otros para sobrevivir. Por eso, dada nuestra configuración antropológica, nuestra ineludible vulnerabilidad y fragilidad, nuestro ser simbólico, necesitamos configurar ámbitos de confianza-o, lo que es lo mismo, relaciones compasivas-, que nos permitan afrontar nuestra condición de seresen-falta ${ }^{18}$.

Sin embargo, Bauman menciona que el hombre de la moderna sociedad líquida es, en definitiva, un sujeto más autónomo pero solitario; pretende interaccionarse, pero eso le ocasiona pánico por lo que pueda implicarle para su condición de liviandad, y su amor por el prójimo, uno de los fundamentos de la vida civilizada y de la moral de Occidente, se ha traducido en temor a los extraños, xenofobia. La variedad cultural que se presenta en el medio urbano globalizado lo aterra por su consecuente ausencia de familiaridad, el resultado es la mixofobia, la inseguridad y el encierro, Bauman menciona que se puede revertir esta deshumanización, enfatizando la necesidad de encontrar nuevos sólidos que persigan un itinerario fijo y un orden estable ${ }^{19}$.

El penetrar en esa intimidad de un contexto hospitalario la persona en estado de embriaguez por medio de la interacción ingresa en el plano de la intersubjetividad, convirtiéndose en una puerta de entrada para ser reconocido como persona.

\section{Conclusiones}

La interacción entre la persona en estado de embriaguez y enfermería durante el cuidado lleva implícito, un intercambio de sentimientos, pensamientos, acciones y experiencias, de las cuales debe surgir una atribución recíproca con el objeto de lograr una intervención terapéutica de calidad humana; pero también de un bienestar por parte de enfermería al brindarlo, en su pensamiento de ambas personas el cuidado es un concepto central y no algo que se designa o impone. El problema reside en intentar rememorar las cualidades fundamentales del cuidado o de lo que el cuidado es y comprende.

La interacción que se lleva a cabo durante el cuidado es un concepto fundamental para entender a la persona en estado de embriaguez, debe tener por lo menos dos partes como requisito, preocupación y ansiedad por la persona, para llegar a esta relación es necesario que la enfermera involucre su propia experiencia para ayudarla a transformarse en una persona fidedigna dentro de la interacción, y pueda ser percibida por la persona en estado de embriaguez como un profesional totalmente involucrado en el cuidado. 
Considerar también el campo de la experiencia de enfermería que se basa fundamentalmente en la acumulación de conocimientos y el desarrollo de las habilidades prácticas, elementos que hasta el momento han sido poco relacionados con la interacción enfermera-persona en estado de embriaguez los cuales tienen un significado importante en la capacitación de la enfermera para responder a cambios particulares en cada situación de cuidado, y aceptar su responsabilidad en las decisiones que toma durante la interacción con la persona en estado de embriaguez.

Si bien, no existe un patrón único de consumo de alcohol, por consiguiente, no existe uniformidad en el cuidado. Cuidar es inseparable de la comprensión y como comprensión debe ser simétrica: escuchando al otro, escuchándonos a nosotros mismos, cuidando del otro y cuidando de nosotros mismos.

En base a lo anterior la interacción durante el cuidado implica esa relación de ambas personas, que a su vez les permite prepararse para la vida cotidiana. El cuidar a la persona en estado de embriaguez debe ser el aspecto más natural y fundamental puesto que ya viene implícito dentro de su propio ser de ambos. En tal sentido enfermería debe desarrollar habilidades para escuchar y responder a las expectativas de la persona en estado de embriaguez considerando su individualidad y características propias, considerando que tiene valor para quien cuida y para quien es cuidado, debiendo ofrecer a ambos la oportunidad de crecimiento.

\section{Bibliografía}

1. Salazar AM, Martínez C. Un sobrevuelo por algunas teorías donde la interacción enfermera-paciente es el núcleo del cuidado. Av. enferm [internet].2008 jul [consultado 17 de mayo 2020]; 26(2):Disponible en: https://revistas.unal.edu.co/index.php/ave nferm/article/view/12945/13541
2. Solano MC. Fenomenología-hermenéutica y enfermería. Cultura de los Cuidados [Internet]. 2006 jun [consultado 01 may 2020]; $62 \quad$ (2): Disponible en: https:/ / rua.ua.es/dspace/bitstream/10045/ 961/1/culturacuidados_19_01.pdf

3. Campos CL, Solis JA. Análisis de la percepción que tienen los infractores de tránsito sobre conducir en estado de embriaguez en la ciudad de Cuenca [bachelor's thesis on the Internet]. [Cuenca]: Universidad de Cuenca; 2016. [cited 18 June 2020]. Available from: http://dspace.ucuenca.edu.ec/handle/1234 $56789 / 23595$

4. Cardozo Y, Escobar CL, Cuartas PA. Interacción del personal de enfermería con el cuidador familiar en el ámbito hospitalario. Investigaciones Andina [Internet]. 2015 [consultado 12 may 2020]; 17(30):12241237. Disponible en: http:/ / www.redalyc.org/articulo.oa?id=239 035878007

5. Getino M. La espera. Construcción social de la muerte en el mundo de los cuidados paliativos. 1a ed. Barcelona, Laertes S.A; 2012.190 p.

6. Rivera O. El enfermo alcohólico: Consideraciones etiológicas terapéuticas. Rev. Medica honduras 1984; 52(4).

7. Kash CR. Habilidades y Competencias en la Interacción Enfermera Paciente. New York: Nurse Red 1986.

8. Lamongtane L. Del aprender al cuidar. Rev Rol Enferm. 1988; 22:79-82.

9. King Imogene. Soins Infermiers. Ottawa, Ed. Du Renoveau, Pedagogique. 1990.

10. Gala FJ, Díaz M, Lupiani M, Bas P, Marchena E. La psicología de la salud en el nuevo curriculum de la diplomatura de enfermería. Rev Rol Enferm, 1995; 206:37-43.

11. Pike AW. Clinical Nature and place or empathy in clinical practice. Philadelphia J. B. Lippincott. 1990. 
12. Bizier M. De la pensé augeste: Sois Infirmiers. Canadá, Decaire 1992.

13. Marriner A, Raile M. Modelos y teorías en enfermería. Madrid: Mosby Doyma. 1994; 418-429.

14. Henderson V. Naturaleza de la enfermería: reflexiones 25 años después. Madrid, Interamericana, Mc Graw-Hill. 1994.

15. Ramírez M, Cárdenas M, Rodríguez S. El Dasein de los cuidados desde la fenomenología hermenéutica de Martín Heidegger. Enferm. univ [Internet]. 2015 Sep [consultado 24 ene 2020]; 12(3): 144-151. Disponible en: http://dx.doi.org/10.1016/j.reu.2015.07.0 03

16. Sánchez A, San Jorge X. Experiencias del personal de enfermería con pacientes hospitalizados por abuso de alcohol. Enferm. univ [internet]. 2017; 14(1):19-27. Disponible en: http://dx.doi.org/10.1016/j.reu.2016.12.0 01

17. López JA, Hernández D, Molina J, Fernández JM, Castaño Al, Sanz Álvarez E, et al. Percepción, conocimientos y actitudes de las enfermeras de urgencias y salud mental frente al alcoholismo y otras drogodependencias. Metas Enferm mar 2014; 17(2): 22-31.

18. Mélich J C. Ética de la compasión. Barcelona: Herder; 2010.317 P.

19. Bauman Z. Tiempos Líquidos. Octava edición. México: Planeta Mexicana, S.A de C.V.2018 